\title{
Artrodese da coluna Lombossacra com o implante A-Systems *
}

\author{
Lumbosacral column arthtodesis with a system's implant
}

Afrane Serdeira'; Tarcísio Eloy Pessoa de Barros Filho², Eduardo Puertas ${ }^{3}$, José Laredo Fillo 4 ,

Rodrigo Di Mare Arbo ${ }^{5}$, AleXAndre M. Glass ${ }^{5}$

RESUMO

Foram avaliados 40 pacientes com espondilolistese e instabilidade lombossacra: 13 pacientes do tipo ístmica-lítica, 19 do tipo degenerativa, 2 pacientes do tipo traumática, 1 tipo do patológica, e 5 do tipo cirúrgica, nos quais foram realizadas 40 artrodeses da coluna lombossacra com enxerto ósseo e fixação metálica com o implante A-Systems.

A avaliação dos pacientes foi realizada clínica e radiograficamente, nos períodos pré-operatório, pós-operatório imediato e pós-operatório tardio. O tempo de acompanhamento clínico máximo foi de 46 meses, o mínimo de 10 meses, obtendo-se um acompanhamento médio de 11 meses.

A avaliação global tanto do ponto vista do paciente como da equipe médica mostrou que: 36 (90\%) dos pacientes obtiveram bom resultado; três pacientes $(7,5 \%)$ apresentaram melhora em relação ao pré-operatório e um paciente (2,5\%) não obteve meIhora. Vinte e nove pacientes $(72,5 \%)$ tiveram sua capacidade de trabalho normalizada, nove $(22,5 \%)$ pacientes tiveram sua capacidade melhorada em 75\% e dois (5\%) pacientes tiveram a capacidade melhorada em $50 \%$.

Vinte e um pacientes (52,5\%) tiveram o índice de Prolo aumentado entre 9 e 10 (excelente); 14 (35\%) tiveram índice de Prolo aumentado para 7 e 8 (bom); em 5 (12,5\%) o índice ficou entre 5 e 6 (regular) e nenhum paciente teve índice 2, 3 ou 4 (mau).

O tempo médio de retorno para as atividades normais variou de três a doze meses, com uma média de sete meses.

Em comparação com métodos similares relatados pela literatura, o implante mostrou-se tão eficiente quanto eles, com as vantagens da simplicidade, da possibilidade de ser usado em vários níveis e da rigidez após montado.

Descritores: Coluna vertebral; Artrodese; Fusão espinal; Implantes artificiais.
SUMMARY

Lumbosacral column arthrodesis with bone graft and metallic fixation (A-Systems implant) was carried out in 40 patients with spondylolisthesis and lumbosacral instability of the following types: isthmic-lytic in 13, degenerative in 19, traumatic in 2, pathological in 1, and surgical in 5.

Preoperative, immediate postoperative, and late postoperative clinical and radiological evaluation was carried out. Clinical follow-up ranged from 10 to 46 months (mean follow-up: 11 months).

Patient's and Physician's overall evaluation showed the following results: good results in 36 (39\%) patients; improvement as compared to preoperative condition in 3 (7.5\%); unchanged condition in 1 (2.5\%); normalized labor capability in 29 (72.5\%) patients; improvement of $75 \%$ in labor capability in 9 (22.5\%) patients; and improvement of $50 \%$ in 2 (5\%) patients. As for the Prolo rate, an increase to 9-10 (excellent) was seen in 21 (52.5\%) patients; an increase to 7 and 8 (good) was seen in 14 (35\%); an increase to 5 to 6 (moderate) was found in 5 (12.5\%) patients. No patient had a Prolo rate of 2, 3, or 4 (poor).

Mean time until return to normal activities ranged from 3 to 12 months (mean: 7 months).

As compared to similar methods reported in literature, the implant has been shown to be equaally effective, with the following advantages: simplicity, use at different levels; rigidity following assembly.

Keywords: Spine; Arthrodesis; Spinal fusion; Prostheses and implants.
*Tese de doutorado (resumo) apresentada ao Programa de Pós-graduação em Ortopedia e Traumatologia da UNIFESP-EPM

1 - Doutor em Medicina. Professor Assistente do Serviço de Ortopedia e Traumatologia do Hospital São Lucas da PUCRS

2 - Professor Associado de Ortopedia e Traumatologia da FMUSP, Chefe de Disciplina de Coluna Vertebral.

3 - Doutor em Medicina, Professor adjunto e Chefe do grupo de Coluna da Disciplina. de Ortopedia e Traumatologia do DOT-UNIFESP-EPM.

4 - Professor Titular do Departamento de Ortopedia e Traumatologia da UNIFESP EPM. Pró-Reitor de Administração da UNIFESP-EPM.

5 - Residente do Serviço de Ortopedia e Traumatologia do Hospital São Lucas da PUCRS

Endereço para correspondência: Serviço de Ortopedia e Traumatologia - Hospital São Lucas PUCRS - Av Ipiranga, 6690 - CEP 90610.000 - Porto Alegre - RS
1.Doctor in Medicine. Assistant Professor at the Orthopedics and Traumatology Department of the São Lucas Hospital of the PUCRS

2.Associate Professor at the Department of Orthopedics and Traumatology of the FMUSP, Head of the Vertebral Spine Discipline

3.Doctor in Medicine, Adjunct Professor and Head of the Column Group of the 3.Doctor in Medicine, Adjunct Professor and Head of the Column Group
Orthopedics and Traumatology Department of the DOT-UNIFESP-EPM. 4.Titular Professor at the Orthopedics and Traumatology Department of the UNIFESP-EPM. Rector of the Administration of UNIFESP-EPM.

5 e 6. Resident Physicians of the Orthopedics and Traumatology Department of São Lucas Hospital of PUCRS

Address : Service of Orthopedics and Traumatology - São Lucas Hospital - PUCRS Av Ipiranga, 6690 CEP 90610-000 Porto Alegre -RS e-mail - afrane@terra.com.b 


\section{INTRODUÇÃO}

Em 1992 iniciamos a pesquisa para confeccionar um implante para fixação da coluna lombossacra que fosse fácil de colocar, não necessitasse de instrumentos complicados, que fosse rígido e seguro. O implante recebeu a denominação genérica de AS1 (Implante A Série 1). Constituía-se de uma haste em U ou duas barras acopladas a grampos e a parafusos pediculares, permitindo uma montagem tridimensional.

Progressivamente introduzimos modificações que aumentaram a resistência e facilitaram a colocação do implante, sem mudar essencialmente o projeto original, recebendo a denominação de A- Systems.

Conseguimos a diminuição do tempo cirúrgico melhorando os encaixes entre os parafusos pediculares e as hastes. Em continuidade à pesquisa mecânica iniciamos o trabalho atual, agora aplicando o método em um estudo clínico-cirúrgico.

\section{MATERIAL E MÉTODOS}

Estudo da casuística:

A casuística deste trabalho é constituída de prontuários de 40 pacientes operados. Os dados foram relacionados segundo o protocolo de Defino(2), modificado, compreendendo os seguintes itens: sexo, idade, diagnóstico, localização da lesão, avaliação da lesão, avaliação ortopédica (dor, mobilidade, força muscular e claudicação), avaliação neurológica (sensibilidade, reflexos e funções esfincterianas) e avaliação por imagem (Rx simples, tomografia computadorizada, avaliação clínica e laboratorial).

A população caracterizou-se por 28 pacientes (70\%) do sexo feminino e 12 (30\%) do sexo masculino com idade variando entre 16 e 76 anos (média de 43,5 anos). Os 40 pacientes apresentavam instabilidade e espondilolistese, sendo agrupados, segundo a Wiltse et al ${ }^{(11)}$, , em 13 pacientes tipo II - A (ístmica - lítica), 19 pacientes tipo III -degenerativa (12 estenoses, 6 degenerações discais e 1 estenose de recesso lateral), 2 do tipo IV (traumática), 1 do tipo V (tumor metastático), 5 do tipo VI (síndrome póslaminectomia). A localização das lesões apresentou maior concentração no nível L5-S1.

Descrição do implante:

O dispositivo de fixação (A-Systems) é composto dos seguintes componentes: (Fig.1)

1. Hastes de aço inoxidável ASTM EF 138, em forma de "U" ou de barras retilíneas, com diâmetro de $6,5 \mathrm{~mm}$ e comprimento variando entre 50 a $150 \mathrm{~mm}$, apresentando superfície recartilhada por usinagem para aumentar o seu coeficiente de atrito;

2. Parafusos pediculares compostos por 3 setores. Iniciando por rosca tipo "dente de serra" com ponta de fresa para osso esponjoso (de $30 \mathrm{~mm}, 35 \mathrm{~mm}, 40 \mathrm{~mm}$ e $45 \mathrm{~mm}$ de comprimento por $5,5 \mathrm{~mm}, 6,5 \mathrm{~mm}$ e $7,5 \mathrm{~mm}$ de diâmetro). Porção intermediária com setor de forma sextavada de $8 \mathrm{~mm}$, seguindo-se com rosca milimétrica de $30 \mathrm{~mm}$ de comprimento por $4 \mathrm{~mm}$ de diâmetro. E finalizando com outra extremidade sextavada de $3 \mathrm{~mm}$ para o encaixe de chave tubular apropriada. Cada parafuso recebe uma porca de $8 \mathrm{~mm}$ e duas arruelas semicilíndricas com face plana de $9 \mathrm{~mm} \times 9 \mathrm{~mm}$;

\section{INTRODUCTION}

In 1992 we started a research to make an implant for lumbosacral column fixation with the following characteristics: easy to use; no need for complicated tools; rigidity and safety. The implant was generically called AS1 (Implant A Series 1). It consisted of a U-shaped shaft or two bars connected to clips and pediculate screws for tridimensional assembly.

Some improvements were progressively implemented to increase resistance and make implant insertion easier, the original project being preserved. The improved version was called A-Systems.

Improved fit of pediculate screws and shafts has led to decreased surgical time. Following mechanical research we started the present study by using the device in a clinical study.

\section{MATERIAL AND METHODS}

\section{Study population}

Fourteen medical records of operated patients were included into the study. The following data according to modified Delfino ${ }^{[2]}$ protocol were recorded: gender, age, diagnosis, lesion site, evaluation of lesion, orthopedic evaluation (pain, mobility, muscular force, and claudication), neurological evaluation (sensitiviy, reflexes, and sphincteral functions), and imaging tests (plain radiographs, computed tomography, clinical and laboratory evaluation).

Twenty-eight female (70\%) and 12 male (30\%) patients aged 16 to 76 years (mean age: 43.5 years) had spondylolisthesis and instability. According to of Wiltse et al. ${ }^{[11]}$, study patients were categorized as follows: type II-A (isthmic-lytic) in 13 patients; type III (degenerative: stenoses in 12; disk degeneration in 6; and stenosis of lateral recess in 1) in 19 patients; type IV (traumatic) in 2; type V (metastatic tumor) in 1; type VI (postlaminectomy syndrome) in 5. Lesions were predominantly found at the L5-S1 level.

\section{Description of the implant:} 1):

A-Systems fixation system consists of the following (Figure

1. U-shaped or straight stainless steel shafts ASTM EF 138 measuring $6.5 \mathrm{~mm}$ diameter and 50 -to- $150 \mathrm{~mm}$ long. Surface is treated to obtain an increased friction coefficient;

2. Pediculate screws consisting of 3 parts: beginning with saw-toothed thread with miller tool for spongy bone (30-mm, 35- $\mathrm{mm}, 40 \mathrm{~mm}$, and 45- $\mathrm{mm}$ long and 5.5- $\mathrm{mm}, 6.5-\mathrm{mm}$, and 7.5$\mathrm{mm}$ diameter). The 8- $\mathrm{mm}$ intermediate portion is hexagon-shaped and followed by millimeter thread measuring $30 \mathrm{~mm}$ long and $4 \mathrm{~mm}$ diameter and ended by another hexagon-shaped end measuring $3 \mathrm{~mm}$ fitting the approriate tubular screwdriver. Each screw has a 8-mm nut and two semicylyndrical washers of plane face measuring $9 \mathrm{~mm} \times 9 \mathrm{~mm}$.

3. Fixation clips (Figure 2) consist of two units measuring 33 $\mathrm{mm}$ long and $10 \mathrm{~mm}$ wide; one end is provided with an oblong opening measuring $12 \mathrm{~mm}$ long and $5 \mathrm{~mm}$ wide to fix pedicula. 
3. Grampos de fixação (Fig.2) constituídos de duas peças de $33 \mathrm{~mm}$ de comprimento por $10 \mathrm{~mm}$ de largura, apresentando numa das extremidades orifício oblongo de $12 \mathrm{~mm}$ de comprimento por $5 \mathrm{~mm}$ de largura para fixar o parafuso pedicular, permitindo deslizamento lateral para a acomodação do mesmo. As bordas do orifício são chanfradas para encaixe das arruelas semi-cilíndricas. O grampo faz a ligação entre a haste e o parafuso e quando estão acoplados obtemos uma montagem tridimensional

4. Ponte estabilizadora: dispositivo preso entre as barras evitando a inclinação lateral após a montagem do sistema quando necessário;

Os instrumentos cirúrgicos:

Os instrumentos para a fixação do implante são (Fig.3): a) iniciador com ponta piramidal, b) guia para pedículo, c) chave tubular sextavada dupla, uma de 8 $\mathrm{mm}$ que se usa externamente para rosquear o parafuso pedicular e a porca sextavada, e uma que se usa internamente de $3 \mathrm{~mm}$ para bloquear o giro do parafuso no aperto final da porca, d) chave sextavada convencional de $8 \mathrm{~mm}$, e) chave de fenda sextavada de $3,5 \mathrm{~mm}$, f) impactor, g) pinça de estiramento e pinça de compressão, h) cortador de barras e modelador de haste.

\section{Técnica cirúrgica:}

Os pacientes, submetidos à anestesia geral, foram posicionados em mesa cirúrgica radiotransparente, em decúbito ventral sobre 4 coxins de espuma de poliuretano revestidos de espuma macia. Aplica-se antisepsia cutânea ea incisão élongitudinal de $10 \mathrm{~cm}$ sobre o segmento lombossacro, após.

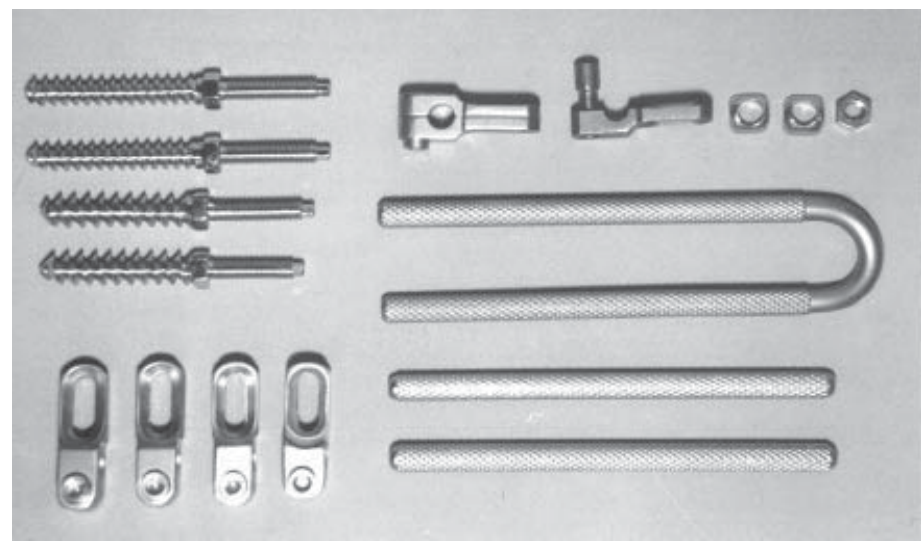

Figura 1 - O implante A-System

Figure 1 - A-System implant

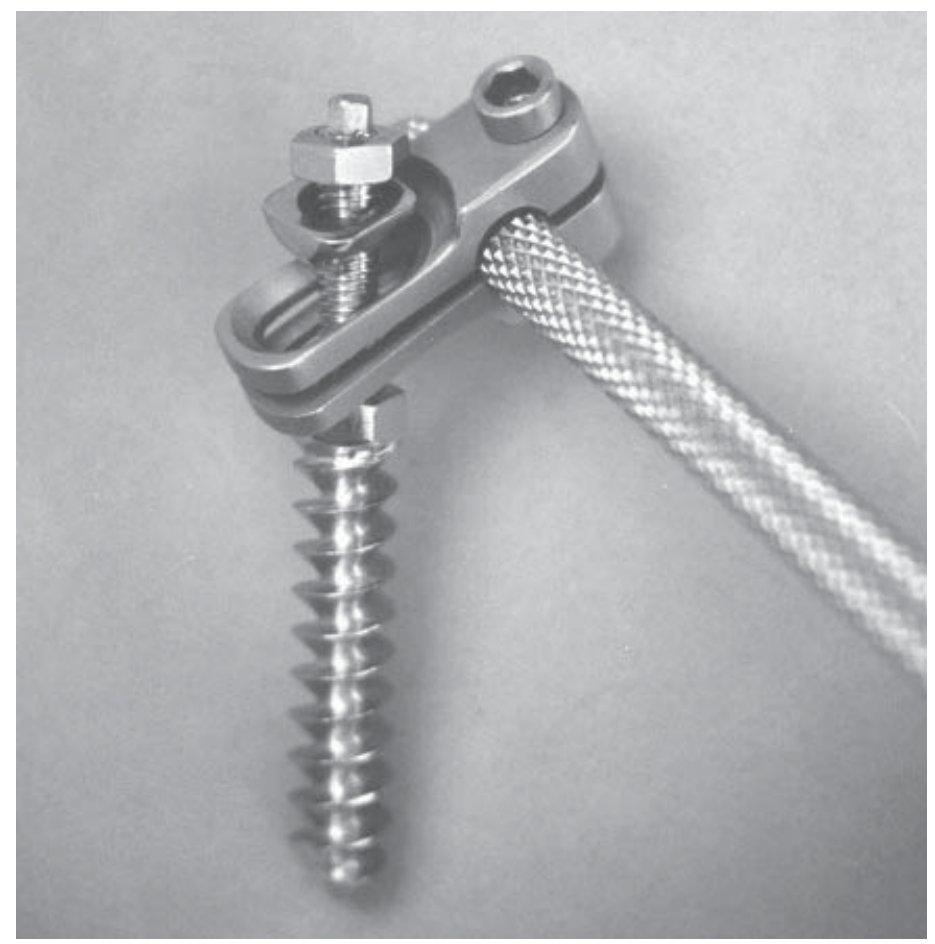

Figura 2 - Grampo de fixação e acoplamento à barra.

Figure 2 - Fixation clip and connection with the bar

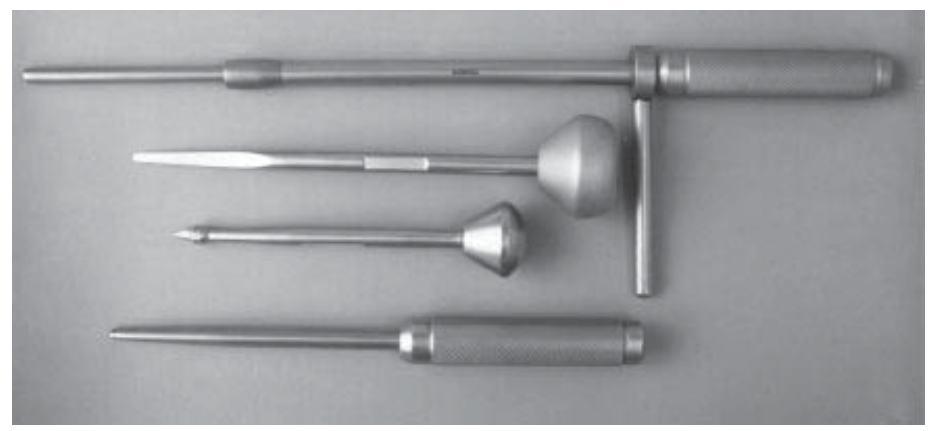

Figura 3 - Instrumentos para colocação do implante. Figure 3 - Tools for implant placement te screw in order that lateral sliding be possible. The clip connects the shaft and the screw. When connected, they form a tridimensional unit.

4. Stabilizer: a device placed between the bars so as to avoid lateral bend following system assembly, as needed.

\section{Surgical tools:}

Tools used for implant fixation are (Figure 3): a) starter with a pyramidal end; b) pedicle guide; c) double hexagon tubular screwdriver, one of which measuring $8 \mathrm{~mm}$ that is used to fit the pediculate screw and hexagon nut; and the other one measuring 3 $\mathrm{mm}$ is internally used to block the screw thread during final turn of the nut; d) a conventional 8-mm hexagon screwdriver; e) 3.5- $\mathrm{mm}$ hexagon slotted screwdriver; f) impactor; g) stretching and compression clampls; h) bar cutter and shaft modeler.

\section{Surgical Technique:}

Patients under general anesthesia were placed in ventral decubitus over 4 polyurethane foam pads covered with soft foam on the radiotransparent operation table. Following skin antisepsis a 10$\mathrm{cm}$ incision was longitudinally made along lumbosacral region.

Fat tissue is dissected so as to expose spinous processes. Paravertebral musculature is subperiosteally detached and supraspinous, interspinal, and ligament flavum. The point corresponding to pedicle is identified before pediculate screw insertion.

This point is identified by crossing two imaginary lines, one of which is transverse and crosses the transverse process; the other line is longitudinal and is approximately 2 $\mathrm{mm}$ distant from the upper articular facet where cortical perforation is made with the sharp pyramidal end. 
Segue-se com divulsão do tecido gorduroso até encontrar os processos espinhosos, descolamento subperiostal da musculatura paravertebral com rugina e remoção do ligamento supraespinhoso, interespinhos e ligamento amarelo. Identificase o ponto correspondente ao pedículo para a introdução dos parafusos pediculares. Este ponto é obtido pela intersecção de duas linhas imaginárias: uma, transversal, que passa sobre o meio do processo transverso e outra, longitudinal, que passa aproximadamente a $2 \mathrm{~mm}$ da faceta articular superior, posição esta onde realiza-se a perfuração cortical com ponta piramidal afiada.

No pedículo usa-se um instrumento-guia que fornece orientação para introduzir o parafuso, permitindo também controlar radiograficamente a orientação e a profundidade desejada .

Procede-se então à fixação dos parafusos (dois para cada nível)). Após este tempo é que praticamos as laminotomias e foraminotomias que se fizerem necessários.

Realiza-se a dissecação da fáscia do glúteo, pela mesma incisão operatória, retirando-se enxertos de osso esponjoso da crista ilíaca com goiva, colocando-se os mesmos entre as apófises espinhosas. Este tempo deve sempre preceder a colocação das hastes e grampos, caso contrário teremos pouco espaço para alojar os enxertos. A inobservância deste detalhe aumenta a incidência de pseudoartroses.

A montagem é feita colocando-se a primeira arruela nos parafusos, com a superfície curva contra o grampo. As hastes e grampos conectores pré-montados são aplicados nos parafusos. Coloca-se a segunda arruela sobre os grampos. A seguir são colocadas as porcas. Após coloca-se dreno de aspiração contínua na crista ilíaca e na área da artrodese, é realizada sutura das fáscias toracolombar e glútea com vicril nº 0 , tecido celular subcutâneo com vicril $n^{\circ} 00$ e pele com poliamida (náilon) $n^{\circ}$ 000 , todos com pontos separados, sendo enfim realizado curativo seco após limpeza da pele com solução fisiológica.

Pós operatório imediato:

No sétimo dia pós-operatório, foram realizadas radiografias do segmento lombossacro nas incidências de frente, perfil, sendo as mesmas comparadas com as imagens obtidas anteriormente.

Avaliação dos resultados:

- Recuperação pós-operatória:

Foram utilizados os seguintes critérios: tempo (número de dias) em que o paciente sentou e deambulou, tempo de hospitalização, período de permanência no hospital, tempo de incapacitação para voltar ao trabalho e tempo de seguimento do paciente.

- Quadro neurológico:

Realizou-se comparação pré e pós-operatória das funções vesicais e intestinais, mobilidade, dor e reflexos.

\section{- Critérios radiográficos:}

As imagens radiográficas do pré e pós-operatório foram analisadas, verificando-se a integração dos enxertos a cada 3 meses, redução dos desvios dos segmentos ósseos, situação do implante e possíveis deslocamentos do mesmo (Fig. 4 ).
The guide is used in the pedicle to guide screw insertion, allowing one to radiographically control direction and deepness.

Screws are then fixed (two for each level). Laminectomies and removal of foramina are then perfomed whenever needed.

Gluteal fascia is dissected through the same incision and spongy bone grafts are removed from the iliac crest. The latter are then inserted between spinous apophyses. This step must always precede shaft and clip insertion. Otherwise, space for grafting will be decreased and pseudarthroses can develop.

Assembly consists of placement of the first washer with the curved face against the clip. Previously assembled shafts and connector clips are applied on screws.

The second washer is put over the clips and nuts are then inserted. Continuous aspiration drainage tube is placed in iliac crest and arthrodesis area, gluteal and toracolumbar fascias are sutured (Vicril \#0), subcutaneous tissue is sutured with vicril \#00, and skin is sutured with nylon \#000 (polyamide).

Separate stitches are used. Finally, a dry dressing is applied following skin cleaning with saline solution.

Immediate postoperative period:

Seven days following surgery lumbosacral radiographs (frontal and lateral views) are obtained and compared with previous radiographs.

\section{Evaluation of Results:}

\section{- Postoperative Recovery:}

The following criteria were used: time (number of days) when the patient sat and walked, hospitalization stay, hospitalization time period, incapacity duration until return to work and followup of the patient.

\section{- Neurological Signs and Symptoms:}

Preoperative and postoperative comparison of vesical and intestinal functions, mobility, pain, and reflexes was carried out.

\section{- Radiological Criteria:}

Pre- and postoperative radiographs were analyzed. The following variables were analyzed: graft integration, decrease in bone shifts, implant localization, and eventual implant displacements (Figure 4).

- Complications:

Complications were reported since their appearance: pain, delayed fracture healing (graft integration after 6 months), pseudarthrosis (lack of healing after 9 months), nervous lesion, bedsore, and infection. 
- Complicações:

As complicações foram relatadas desde o seu aparecimento: dor, retardo da consolidação (integração dos enxertos após 6 meses), pseudoartrose (ausência de consolidação após 9 meses), lesão nervosa, escara e infecção.

- Situação do implante:

A situação do implante foi avaliada pela análise das radiografias pós-operatórias observando-se os seguintes pontos: sem problemas, parafusos soltos, parafusos quebrados, porcas soltas, grampos soltos, hastes quebradas, arames quebrados e falhas da interface parafuso-osso.

Avaliação final:

Avaliação objetiva: foi adotada a classificação quantitativa pelo índice de Prolo(5) $^{(5)}$ que avalia o estado laborativo (econômico) e funcional (dor) (Quadro 1).

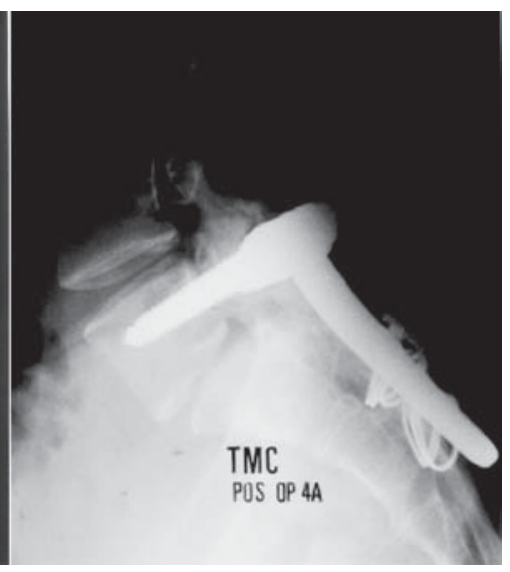

Figura 8 - Rx pré e pós-operatório

Figure 4 - Preoperative and postoperative radiographs

\begin{tabular}{|c|c|c|c|}
\hline $\begin{array}{l}\text { ECONOMICA (Produtividade) Economic } \\
\text { (Productivity) }\end{array}$ & & & $\begin{array}{l}\text { FUNCIONAL (Dor) Functional } \\
\text { (Pain) }\end{array}$ \\
\hline Fullly disabled Inválido completo & E1 & F1 & $\begin{array}{l}\text { Incapaz total (pior que antes) } \\
\text { Fully disabled (worse than } \\
\text { before) }\end{array}$ \\
\hline $\begin{array}{l}\text { With no profitable activities (including home } \\
\text { activities) Sem ocup. lucrativas (incluindo } \\
\text { ativ. do lar) }\end{array}$ & E2 & F2 & Moderate to severe pain Dor \\
\hline $\begin{array}{l}\text { Trabalh. ativo, não como no pré-morbido } \\
\text { Active work, not similar to that before } \\
\text { disease }\end{array}$ & E3 & F3 & $\begin{array}{l}\text { Mild pain daily (better) } \\
\text { Baixo nivel de dor diária (melhor) }\end{array}$ \\
\hline $\begin{array}{l}\text { Trabalhando ativo com limitações Active } \\
\text { work with limitations }\end{array}$ & E4 & $\mathrm{F} 4$ & $\begin{array}{l}\text { With no pain or with occasional } \\
\text { pain Sem dor ou dor ocasional }\end{array}$ \\
\hline $\begin{array}{l}\text { Trabalhando ativo sem limitaçōesActive } \\
\text { work without limitations }\end{array}$ & E5 & F5 & $\begin{array}{l}\text { Sem dor, voltou às atividades } \\
\text { With no pain, return to activities }\end{array}$ \\
\hline
\end{tabular}

Quadro 1: ESCALA DE PROLO: AVALIAÇÃO ECONÔMICA E FUNCIONAL

Charlt 1 : PROLO SCALE: ECONOMIC AND FUNCTIONAL EVALUATION

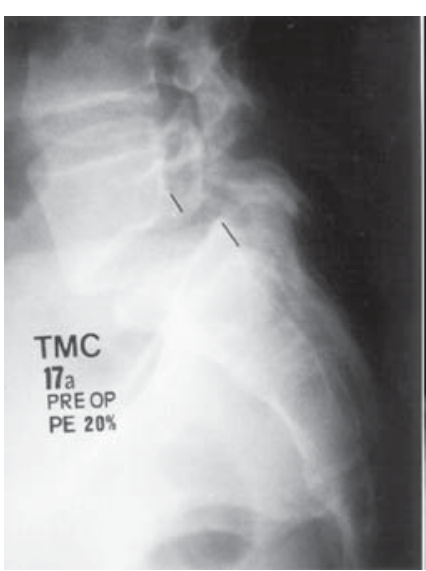

- Implant status:

Implant status was evaluated by analyzing postoperative radiographs based upon the following points: no problem, loose screws, broken screws, loose nuts, loose clips, broken shafts, broken wire, and defects in screw-bone interface.

\section{- Final Evaluation:}

Objective evaluation: the quantitative classification was adopted using Prolo rate ${ }^{[5]}$ that evaluates labor (economical) status and functional status (pain) (Chart 1).

Subjective Evaluation: patient's and physician's opinion was taken into account according to the following categories: good, improved, unaltered, worse; and 100\%, 75\%, 50\%, 25\% labor capability; and total disability.

- Statistical Analysis:

Descriptive statistical analyses of quantitative variables and absolute and relative frequency distribution (\%) of qualitative variables was performed. A significance level of 0.05 was adopted and significant results were marked with an asterisk $\left({ }^{*}\right)$., e do médico, em bom, melhor, inalterado e pior, e capacidade para o trabalho em 100\%, 75\%, 50\%, 25\% e incapaz total.

\section{- Análise estatística:}

Realizou-se estatística descritiva das variáveis quantitativas e distribuição das freqüências absolutas e relativas (\%) das variáveis qualitativas adotamos o nível de significância 0,05 e resultado de significância com asterisco $\left(^{*}\right)$.

\section{RESULTADOS}

Análise clínica:

- Quadro neurológico:

a) Funções:

As funções vesical e intestinal permaneceram alteradas no paciente que teve fratura por explosão. Nos outros pacientes não ocorreram alterações nestas funções.

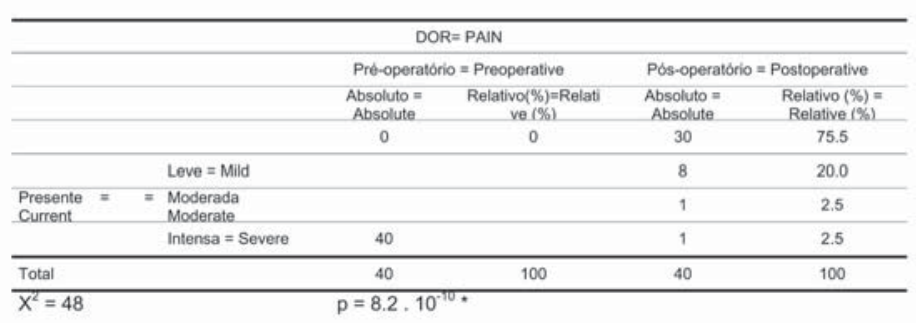

Tabela 1 - Distribuição de freqüência absoluta e relativa (\%) da ocorrência de dor pré e pós-operatórias. Comparação pelo teste Qui quadrado $(\mu=0,05)$

Table 1 - Absolut and relative (\%) frequency distribuition and occurrence of pre and postoperative pain. Comparison by square qui test $(\mu=0,05)$.
- Neurological Signs and Symptoms:

\section{a) Functions:}

Vesical and intestinal functions remained altered in the patient who had an explosion fracture. No changes were observed in these functions in the remaining patients.

\section{b) Pain and Sensitivity:}

Following surgery pain totally subsided in 30 patients (75\%) (Table 1). 
b) Dor e sensibilidade:

Segundo a tabela 1, no pós-operatório 30 pacientes (75\%) tiveram alívio total da dor.

c) Reflexos (Tabela 2 e 3):

- Mobilidade (Tabela 4):

Avaliação funcional:

- Capacidade de trabalho (tabela 5):

- Tempo de incapacidade para o trabalho:

Quatro pacientes (10\%) voltaram ao trabalho entre 3 e 4 meses, 15 pacientes $(37,5 \%)$ entre 3 e 5 meses, 18 pacientes (45\%) entre 5 e 7 meses e 3 pacientes $(7,5 \%)$ ultrapassaram 12 meses.

- Situação do implante:

Trinta e oito implantes (95\%) permaneceram sem problemas até o momento final deste estudo, em 2 (5\%) houve afrouxamento de um grampo por falha técnica. Não foram constatadas quebras dos parafusos ou hastes.

- Tempo de acompanhamento pós-operatório:

O temo máximo foi de 46 meses e mínimo de 10 meses, com uma média de 11 meses.

\section{- Complicações:}

Observamos 11 complicações $(27,5 \%)$, sendo 5 inerentes à fixação do implante: 2 à pseudoartrose, 2 ao retardo de consolidação do enxerto e 1 à escara e infecção secundária de partes moles na região sacra por falha técnica. As outras 6 não tiveram relação com o implante: 4 devido à dor na área doadora do enxerto ósseo por lesão dos nervos clúneos, 1 à dor transitória na espinha ilíaca anterior e 1 à permanência da mesma dor lombar.

- Resultados finais;

Considerou-se que 36 (90\%) dos pacientes obtiveram bom resultado. Em 3 (7,5\%) obtivemos melhora em relação ao pré-operatório e 1 (2,5\%) não retornou, permanecendo inalterado (abandono de trata-

\begin{tabular}{|c|c|c|c|c|c|c|c|c|}
\hline \multicolumn{9}{|c|}{ Patellar reflex Reflexo patelar ( 80 ) } \\
\hline & \multicolumn{4}{|c|}{ Prè-operatório $=$ Preoperative } & \multicolumn{4}{|c|}{ Pós-operatório = Postoperative } \\
\hline & $D=R$ & $E=L$ & $\begin{array}{c}\text { Absoluto } \\
= \\
\text { Absolute }\end{array}$ & $\begin{array}{c}\text { Relativo(\%) } \\
=\text { Relative } \\
(\%)\end{array}$ & $D=R$ & $E=L$ & $\begin{array}{c}\text { Absoluto } \\
= \\
\text { Absolute }\end{array}$ & $\begin{array}{c}\text { Relativo(\%) } \\
=\text { Relative } \\
(\%)\end{array}$ \\
\hline Normal & 23 & 22 & 45 & 56.25 & 36 & 36 & 72 & 90 \\
\hline Altered & 26 & 9 & 35 & 43.75 & 4 & 4 & 8 & 10 \\
\hline Total & 40 & 40 & 80 & 100.0 & 40 & 40 & 80 & 100.00 \\
\hline
\end{tabular}

Tabela 2 - Distribuição de freqüência absoluta e relativa (\%) da ocorrência do reflexo patelar pré e pós-operatórios. Comparação pelo teste Qui quadrado $(\mu=0,05)$.

Table 2 - Absolut and relative (\%) frequency distribuition and occurrence of pre and postoperative patellar reflex. Comparison by

\begin{tabular}{|c|c|c|c|c|c|c|c|c|}
\hline \multicolumn{9}{|c|}{ Reflexo aquileu $(80)=$ Achilles tendon reflex } \\
\hline & \multicolumn{4}{|c|}{ Pré-operatório $=$ Preoperative } & \multicolumn{4}{|c|}{ Pós-operatório = Postoperative } \\
\hline & $D=R$ & $E=L$ & $\begin{array}{c}\text { Absoluto } \\
= \\
\text { Absolute }\end{array}$ & $\begin{array}{c}\text { Relativo(\%) } \\
=\text { Relative } \\
(\%)\end{array}$ & $D=R$ & $E=L$ & $\begin{array}{c}\text { Absoluto } \\
= \\
\text { Absolute }\end{array}$ & $\begin{array}{c}\text { Relativo(\%) } \\
=\text { Relative } \\
(\%)\end{array}$ \\
\hline Normal & 19 & 19 & 38 & 47,5 & 34 & 34 & 68 & 85,0 \\
\hline Altered & 21 & 21 & 42 & 52,5 & 6 & 6 & 12 & 15,0 \\
\hline Total & 40 & 40 & 80 & 100,0 & 40 & 40 & 80 & 100,00 \\
\hline
\end{tabular}

Tabela 3 - Distribuição de freqüência absoluta e relativa (\%) da ocorrência do reflexo aquileu pré e pós operatórios. Comparação pelo teste Qui quadrado $(\mu=0,05)$.

Table 3 - Absolut and relative (\%) frequency distribuition and occurrence of pre and postoperative achilles reflex. Comparison by square qui test $(\mu=0,05)$.

\begin{tabular}{lcccc}
\hline \multicolumn{5}{c}{ Mobilidade da coluna $=$ Spine Mobility } \\
\hline & $\begin{array}{c}\text { Pré-operatória }=\text { Preoperative } \\
\text { Absoluto }=\end{array}$ & $\begin{array}{c}\text { Relativo }(\%)= \\
\text { Relative }(\%)\end{array}$ & $\begin{array}{c}\text { Absolute }= \\
\text { Absolute }\end{array}$ & $\begin{array}{c}\text { Relativo }(\%)= \\
\text { Relative }(\%)\end{array}$ \\
\hline Normal $=$ Normal & 3 & 7,5 & 30 & 75,0 \\
\hline $\begin{array}{l}\text { Diminuida }= \\
\text { Decreased }\end{array}$ & 37 & 92,5 & 10 & 25,0 \\
\hline Total & 40 & 100,0 & 40 & 100,00 \\
\hline $\mathrm{X}^{2}=37.6$ & $\mathrm{P}=0.0^{\circ}$ & & &
\end{tabular}

Tabela 4 - Distribuição de freqüência absoluta e relativa (\%) da ocorrência da mobilidade da coluna pré e pós operatória. Comparação pelo teste Qui quadrado $(\mu=0,05)$.

Table 4 - Absolut and relative (\%) frequency distribuition and occurrence of pre and postoperative spine mobility. Comparison by square qui test $(\mu=0,05)$.

\begin{tabular}{lcccc}
\hline \multicolumn{5}{c}{ Mobilidade da coluna = Spine mobility } \\
\hline & Pré-operatória $=$ Before surgery & Pós-operatória = After surgery \\
\hline $\begin{array}{l}\text { Insatisfatório Not } \\
\text { satisfactory }\end{array}$ & $\mathbf{3 9}$ & $\mathbf{9 7 . 5}$ & $\mathbf{5}$ & $\mathbf{1 2 . 5}$ \\
\hline Mau (2,3 e 4) Poor & 20 & 50.0 & 0 & 0 \\
\hline $\begin{array}{l}\text { Regular (5 e 6) } \\
\text { Modest }\end{array}$ & 19 & 47.5 & 5 & 12.5 \\
\hline $\begin{array}{l}\text { Satisfatório } \\
\text { Satisfactory }\end{array}$ & $\mathbf{1}$ & $\mathbf{2 . 5}$ & $\mathbf{3 5}$ & $\mathbf{8 7 . 5}$ \\
\hline Bom ( 7 e 8) Good & 1 & 2.5 & 14 & 35.0 \\
\hline $\begin{array}{l}\text { Excelente (9 e 10) } \\
\text { Excellent }\end{array}$ & 0 & 0.0 & 21 & 52.5 \\
\hline Total & $\mathbf{4 0}$ & $\mathbf{1 0 0 . 0}$ & $\mathbf{4 0}$ & $\mathbf{1 0 0 . 0}$ \\
\hline $\mathrm{X}^{2}=60.43$ & $\mathrm{P}=1.6 .10^{10-0.0}$ & & &
\end{tabular}

Tabela 5 - Distribuição de freqüência absoluta e relativa (\%) dos pacientes obtidas segundo a classificação de Prolo no pré e pós operatórios. Comparação pelo teste do Qui quadrado $(\mu=0,05)$.

Table 5 - Absolut and relative (\%) frequency distribuition of patients from pre and postoperative Prolo classification. Comparison by square qui test $(\mu=0,05)$. c) Reflexes (Tables 2 and 3):

- Mobility (Table 4):

Functional Evaluation:

- Labor capability (Table 5):

- Labor incapacity duration:

Four patients (10\%) returned to work within 3 to 4 months; 15 patients (37.5\%) returned to work within 3 to 5 months; 18 patients (45\%) returned to work within 5 to 7 months; and 3 patients (7.5\%) returned to work within more than 12 months.

- Implant status:

Thirty-eight implants (95\%) had no complications until study conclusion; in 2 patients (5\%) loose clip developed due to faulty technique. Breakage of screws and shafts were not observed.

- Postoperative followup:

Postoperative follow-up ranged from 10 to 46 months (mean: 11 months).

\section{- Complications:}

Eleven complications (27.5\%) developed: 5 associated with implant fixation; 2 associated with pseudarthrosis; 2 associated with delayed graft integration; and 1 associated with sacral bedsore and secondary infection due to faulty technique. The other six complications were not related with the implant: 4 were due to pain in the bone graft donor area secondary to rami cluneum 
mento). Estas afirmações baseiam-se no estudo objetivo das planilhas, no grau de satisfação do paciente e pela opinião da equipe médica envolvida no tratamento (Fig.9).

\section{DISCUSSÃO}

Aplicando-se protocolos semelhantes a outros trabalhos constantes na literatura, foram estudados os prontuários de 40 pacientes submetidos ao tratamento cirúrgico para fixação do segmento lombossacro da coluna vertebral que não obtiveram alívio com tratamento conservador. Na análise dos dados destes pacientes, a distribuição dos sintomas e sinais por idade, que incidiu entre 16 e 76 anos, configura achado comparável com os trabalhos publicados por Rissanen ${ }^{(6)}$ Twoney e Taylor $^{(9)}$, Frymoyer ${ }^{(3)}$ e Barros Fo et al. (1). Quanto ao sexo, 28 (70\%) eram do sexo feminino e 12 (30\%) do sexo masculino, sendo que em Rosenberg ${ }^{(7)}$, o sexo feminino apresenta incidência de espondilolistese dege-nerativa 6 vezes maior.

Todos os pacientes eram portadores de instabilidade, pelas evidências observadas na avaliação pré-operatória ${ }^{(10)}$, intra-operatoriamente por visão direta e confirmada após a descompressão vertebral necessária dos elementos posteriores (arcos neurais e processos articulares) de acordo com Kanwaldeep e Herkowitz ${ }^{(4)}$.

Verificamos que todos os pacientes queixavam-se de dor intensa e sensibilidade alterada com predominância de lesão na raiz L5, nos quais obtivemos melhora evidente no pós-operatório.

Pela análise dos dados obtidos nas tabelas, os reflexos meIhoraram significativamente no pós-operatório.

A mobilidade voltou à normalidade em 30 pacientes (75\%) e 10 ficaram com diminuição leve da mesma (25\%). A amplitude dos movimentos em 30 pacientes voltou ao normal (75\%), em 9 permaneceu diminuída e 1 obteve discreta melhora (25\%). A melhora da mobilidade constitui-se em um fator muito positivo para a qualidade de vida dos pacientes. Vinte e nove pacientes lesion; one was due to transient pain in the anterior iliac crest; and 1 was due to lumbar pain persistence.

\section{- Final results}

Good results were observed in 36 (90\%) patients. Improvement as compared to preoperative status was seen in $3(7.5 \%)$ patients and 1 (2.5\%) patient whose condition was considered unaltered did not return (lost to follow-up), . These results are based upon the objective study of medical records, patient's satisfaction, and physician's opinion (Figure 5).

\section{DISCUSSION}

Medical records of 40 patients were studied according to similar protocols described in literature.

All patients were submitted to surgical therapy for lumbosacral stabilization following failure of conservative therapy.

Data concerning distribution of signs and symptoms according to age (range: 16-76 years) are similar to those reported by Rissanen [6], Twoney and Taylor [9], Frymoyer ${ }^{[3]}$, Barros $F^{\circ}$ et al. and Basile [1].

In contrast, data concerning gender were not similar: in our studies female:male ratio was $28: 12$ (70\%:30\%) while Rosenberg ${ }^{[7]}$ has reported that the incidence of degenerative spondylolisthesis is 6 times greater in female patients

All patients had instability, as shown by evidence found in preoperative evaluation [10] and by direct examination during surgery. This finding was confirmed after vertebral decompression of posterior elements (neural arches and articular processes) according to Kanwaldeep and Herkowitz ${ }^{[4]}$.

All patients had severe pain and sensitivity changes with predominance of L5-root lesion. Improvement was evident following surgey. 
(72,5\%) tiveram sua capacidade de trabalho normalizada, 9 (22,5\%) tiveram sua capacidade melhorada em $75 \%$ e 2 (5\%) ficaram com a capacidade melhorada em 50\%. Esta avaliação é baseada na opinião do paciente e do médico, podendo este dado sofrer alguma distorção subjetiva. Para minimizar estas distorções, nos baseamos na classificação de Prolo et al. ${ }^{(5)}$.

Comparando-se o pré e o pós-operatório observou-se que o índice de Prolo aumentou, traduzindo-se por melhora significativa. A soma do grau econômico e funcional nos fornece um resultado que define o estado clínico do paciente na seguinte forma: 21 pacientes (52,5\%) tiveram o índice de Prolo aumentado entre 9 e 10 (excelente), 14 (35\%) tiveram o índice aumentado para 7 e 8 (bom), $5(12,5 \%)$ ficaram entre 5 e 6 (regular) e nenhum teve índices 2, 3 e 4 (mau), (Figuras, $5 a$ e 5b).

Analisando os implantes verificamos que 38 (95\%) permaneceram sem problemas até o momento final da pesquisa.

Em 2 implantes (5\%) houve afrouxamento de um grampo por falha técnica. Não foram observadas quebras de parafusos ou hastes.

Tendo em vista a grande diversidade de implantes e de métodos de fixação da coluna existentes atualmente, e estando estes métodos em constante evolução, pensamos que o importante é possuir experiência com um sistema, de fácil manejo e embasado em pesquisa científica. O cirurgião deve estar ciente das peculiaridades técnicas do implante, das vantagens, das dificuldades e dos cuidados que o seu uso requer. Baseado nos resultados dos estudos mecânico(8) acredita que o sistema de implante A-Systems é adequado e passamos a usá-lo, comprovando-se neste estudo a sua eficiência.
Based upon data shown in tables, reflexes significantly improved following surgery.

Painful mobility returned to normal in 30 patients (75\%) while mildly decreased painful mobility remained in 10 patients (25\%). Motion range returned to normal in 30 patients (75\%), remained decreased in 9, and slightly improved in 1 (25\%).

Mobility improvement is a very positive factor for patient's quality of life. In 29 patients (72.5\%) labor capability returned to normal, had a $75 \%$ improvement in 9 (22.5\%) and 50\% improvement in 2 (5\%).

This evaluation is based upon patient's and physician's opinion and is subject to some subjective distortion. The classification described by Prolo et al. ${ }^{[5]}$ was used so as to reduce distortions.

The Prolo rate increased when preoperative and postoperative values were compared, thus confirming a significant improvement.

The sum of the economic and functional degrees provides a result defining patient's clinical condition as follows: an increase to 9-10 (excellent) was seen in 21 (52.5\%) patients; an increase to 7 and 8 (good) was seen in 14 (35\%); an increase to 5 to 6 (moderate) was found in 5 (12.5\%) patients. No patient had a Prolo rate of 2, 3, or 4 (poor).

Implant analysis showed no complication in 38 (95\%) patients until study conclusion. Loose clip was seen in 2 patients (5\%) due to faulty technique. Screw or shaft breakages were not seen.

Considering the great diversity of implants and fixation methods available today and constant improvements of these resources, we concluded that an important point is the experience with an easy-to-use system validated by scientific research.

The surgeon must be aware of technical peculiarities of the implant, advantages, difficulties, and required care for its use. 


\section{CONCLUSÕES:}

1. O fixador interno A-Systems está indicado para tratamento cirúrgico de patologias da coluna lombossacra que requeiram artrodese;

2. Pode ser usado na face posterior dos corpos vertebrais do segmento lombossacro da coluna vertebral;

3. Possibilita artrodeses curtas pois o método se baseia na sustentação pedicular;

4. Mesmo quando usados amarrilhos no sacro e fixação pedicular lombar, os resultados finais foram bons;

5. Permite mobilização precoce do paciente, com índice baixo de falhas mecânicas e de pseudo-artrose;

6. O índice de complicações não é maior do que os observados em séries semelhantes descritas na literatura.
Based upon mechanical study results ${ }^{[8]}$, the A-Systems implant system is adequate. We adopted it and the present study confirmed its efficacy.

\section{CONCLUSIONS:}

1. The inner fixator A-Systems is indicated for surgical treatment of lumbosacral column diseases requiring arthrodesis.

2. It can be used in the posterior side of vertebral bodies of the lumbosacral column.

3. It allows one to perform short arthrodesis since the method is based upon pediculate support;

4. Final results were good even when lumbar pediculate fixation or sacral threads were used.

5. Early mobilization of patients is possible with a low rate of mechanical failure and pseudarthrosis.

6. The complication rate is not greater than those seen in similar series described in literature.

\section{REFERÊNCIAS BIBLIOGRÁFICAS}

1. Barros Filho TEP, Camargo OP, Oliveira RP, Croci AT, Prada FS. Avaliação e planejamento cirúrgico dos tumores metastáticos da coluna vertebral. Rev Bras Ortop 28:788-790, 1993

2. Defino HLA. Utilização do sistema U.S.I.S. na fixação da coluna vertebral [Livre-Docência]. Ribeirão Preto: Faculdade de Medicina de Ribeirão Preto, 1995.

3. Frymoyer JW. Segmental instability: overview and classification. In: Frymoyer JW. The adult spine: principles and practice. New York: Raven Press, 1991 p.1873-1891.

4. Kanwaldeep SS, Herkowitz HN. Spinal instrumentation in the management of degenerative disorders of the lumbar spine. Clin Orthop 355:39-53, 1997.

5. Prolo JD, Oklund AS, Butcher M. Toward uniformity in evaluating results of spine operation. A paradigm applied to posterior lumbar interbody fusions. Spine 11:601-606, 1986

6. Rissanen PM. Comparison of pathological changes in intervertebral disc and interspinous ligaments of the lower part of the lumbar spine in the light of autopsy findings. Acta Orthop Scand 34:54-65, 1964.
7. Rosenberg NJ. Degenerative spondylolisthesis: surgical treatment. Clin Orthop 117:112-120, 1976.

8. Serdeira A. Estudo mecânico de implante para fixação do segmento lombossacro da coluna vertebral. [Dissertação]. São Paulo: Escola Paulista de Medicina, 1994.

9. Twomey L, Taylor J. Flexion creep deformation and hysteresis in the lumbar vertebral column. Spine 2:116-122, 1982.

10. White AA, Panjabi MM. The problem of clinical instability in the human spine: A sistematic approach. In: White AA. Clinical biomechanics of the spine. Philadephia: Lippincott, 1978. p.192-276.

11. Wiltse LL, Bateman JG, Hutchinson NR, Nelson WE. The paraspinal sacrospinalis-splitting approach to the lumbar spine. J Bone Joint Surg Am 50:919926, 1968 